\title{
Dynamic Response of a Thyristor Controlled Switched Capacitor
}

$\begin{array}{ccc}\text { Sasan G. Jalali } & \text { Robert H. Lasseter } & \text { Ian Dobson } \\ \text { Student Member } & \text { Fellow } & \text { Member }\end{array}$

Electrical and Computer Engineering Department

University of Wisconsin, Madison, WI 53706

\begin{abstract}
This paper computes the small signal dynamic response of a thyristor controlled series capacitor system for use in control design. The computation includes the effects of synchronization and the nonlinearity due to thyristor switchings. Eigenvalues of the small signal dynamic response are computed and used to study the dynamic response of the Kayenta system using different methods of synchronization and a closed loop control.
\end{abstract}

Keywords: FACTS, TCSC, Kayenta, TCR.

\section{Introduction}

The optimization of controllers for a Thyristor Controlled Series Capacitor (TCSC) is critical for its use in power systems. This is a difficult task due to the nonlinearities introduced by the switching of the thyristors. In particular, the dynamic response of a TCSC changes as a function of its operating point. This dependence is discussed in papers describing the design of the Kayenta system $[1,2]$.

\begin{tabular}{cc} 
Table 1. Complex poles for Kayenta System [1] \\
\hline$\sigma=20^{\circ}$ & $s=-14.5 \pm j 56.2$ \\
$\sigma=40^{\circ}$ & $s=-13.5 \pm j 48.1$ \\
$\sigma=50^{\circ}$ & $s=-10.6 \pm j 30.2$ \\
$\sigma=60^{\circ}$ & $s=-13.5 \pm j 11.9$ \\
\hline
\end{tabular}

The design of the Kayenta controller was achieved by first finding the open loop response of line current to changes in the firing point using a detailed EMTP model. The resulting envelope of the line current response enabled control engineers to find a transfer function which approximates the dynamics of the system around an operating point. The resulting fourth order transfer function has two poles on the real axis and a complex pair of poles. The dependence of the complex poles on the operating point is shown in Table 1. The operating points are specified by $\sigma$, the thyristor conduction time in electri-

94 WM 065-3 PWRD A paper recommended and approved by the IEEE Transmission and Distribution Committee of the IEEE Power Engineering Society for presentat. Ion at the IEEE/PES 1994 Winter Meeting, New York New York, January 30 - February 3, 1994. Manuscript submitted August 2, 1993; made available for printing December 6, 1993. cal degrees. The natural frequencies of oscillation range from $8.9 \mathrm{~Hz}$ to $1.9 \mathrm{~Hz}$.

The control design for Kayenta was achieved by using these transfer functions in a closed loop model allowing the use of standard tools to optimize the control response. This method is effective but provides little insight into the behavior of the system and requires detailed EMTP simulations to find the necessary transfer functions. This paper presents a first principles method for computing eigenvalues of the small signal response of TCSC systems. The method takes account of the nonlinearities of the thyristor switchings and the synchronization and controller dynamics. Related work includes computations of the closed loop dynamic response of controlled AC/DC or resonant convertors $[3,4,5]$ and analysis of instabilities, damping, and resonance in an open loop static Var control system $[6,7,8]$.

\section{Kayenta System}

The $230 \mathrm{kV}, 330$ Mvar TCSC system shown in Figure 1 was installed in northeastern Arizona at the Kayenta Substation. There are two conventional 165 Mvar series capacitor banks with a nominal reactance of $55 \Omega$ each. One of these segments is divided into $40 \Omega$ and $15 \Omega$. The $15 \Omega$ unit is configured as a TCSC so that $15 \Omega$ of capacitance is in parallel with a Thyristor Controlled Reactor (TCR) of $2.56 \Omega$.

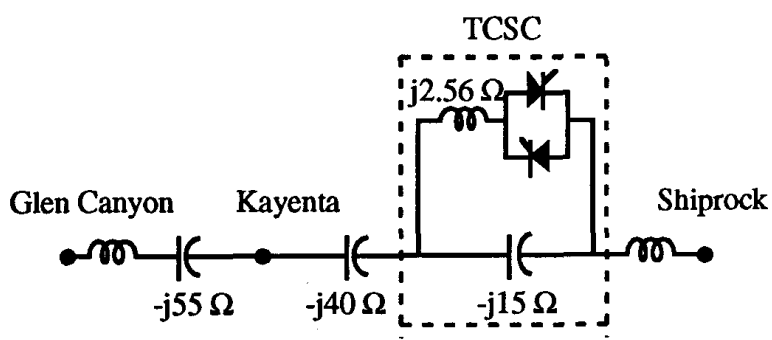

Figure 1. Kayenta System

The fundamental impedance of the TCSC is similar to that of a parallel LC circuit with a variable inductance. With the thyristors off and conduction time $\sigma=0^{\circ}$, the impedance per phase is $15 \Omega$. As $\sigma$ is increased, the capacitance increases to a large value at the fundamental resonance point $\sigma=74^{\circ}[1]$. It is assumed that control limits prevent even temporary operation near the resonance. It is also assumed that operation will be limited to the capacitive region except for full bypass at $\sigma=180^{\circ}$. 
Basic Concepts of TCSC Dynamics

The dynamics of any system which is periodic with period $T$ can be studied by sampling the system states once per period. If the resulting values of the state variables are equal to the previous sampled states, the system is in steady state. The system dynamics can be described as the change in the sampled states from one sampled point to the next. This concept is formalized as the Poincaré mapping $F$ from dynamical systems theory [9] which maps the system states forward in time from $t_{0}$ to $t_{0}+T$. That is, $F\left[x\left(t_{0}\right)\right]=x\left(t_{0}+T\right)$ where $x$ represents the system states. If $F\left[x\left(t_{0}\right)\right]=x\left(t_{0}\right)$, the map has a fixed point $x\left(t_{0}\right)$ and the system is in steady state.

Figure 2 describes the system dynamics as the TCSC states evolve over a period $\mathrm{T}$. The conduction periods starting at times $\phi_{0}, \phi_{1 / 2}, \phi_{1}$ are defined by the control system and the method used to synchronize the thyristor firing times to the system states. The turn off times $\tau_{0}, \tau_{1 / 2}, \tau_{1}$ are defined as the times at which the thyristor current becomes zero. The dependence of these switching times on the system states causes the system nonlinearity.

At the beginning of the period $t_{0}$, the initial states are the vector $x_{0}$ and a thyristor is on. This conduction mode ends when the thyristor current goes through zero at $\tau_{0}$. The off or non-conducting mode starts at $\tau_{0}$ and continues until the next firing pulse is applied at $\phi_{1 / 2}$, where the subscript $1 / 2$ refers to the half period. The system progresses through a thyristor firing at time $\phi_{1 / 2}$, a thyristor turn off at $\tau_{1 / 2}$ and a thyristor firing at $\phi_{1}$ until arriving at the end of the period at $t_{0}+T$ with states $F\left(x_{0}\right)$. The Poincaré map $F\left(x_{0}\right)$ takes into account the dependence of the switching times on the system states and can be computed by integrating over the period the appropriate state equations as determined by the switching times.

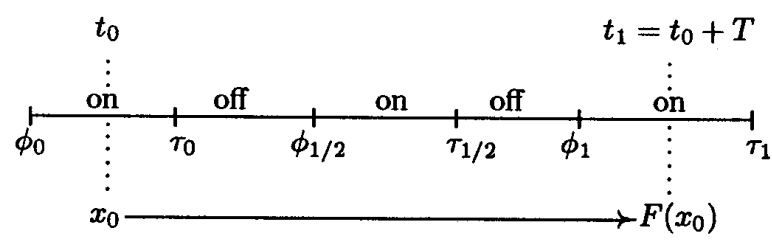

Figure 2. System dynamics over one period.

The small signal stability of the TCSC can now be computed from the Jacobian of the Poincaré map evaluated at a sampled steady state $x_{0}$.

$$
\text { Jacobian }=D F\left(x_{0}\right)
$$

The detailed derivation of the Poincaré map and its Jacobian is given in Appendices B and C. The eigenvalues of the Jacobian $D F$ provide information on the small signal dynamics of the system. More precisely, the eigenvalues are the poles of the sampled data transfer function which is the best linear approximation to the system response to small disturbances. In particular, the steady state is exponentially stable if the eigenvalues of the Jacobian evaluated at $x_{0}$ lie strictly inside the unit circle. The eigenvalues or poles lie in the $z$-plane familiar from sampled data systems. The expected relationship between $s$-plane and $z$-plane poles applies so that an $s$-plane pole $s=-a \pm j \omega$ maps to the $z$-plane as $z=e^{-a T} e^{ \pm j \omega T}$. The magnitude $|z|=e^{-a T}$ defines the damping so that a pole inside the unit circle has positive damping and a pole outside the unit circle implies instability. The polar angle, $\omega T$ defines the frequency of oscillation about the sampling frequency. As polar angle traverses from $0^{\circ}$ to $360^{\circ}$ the modulation frequency increases from zero to the sampling frequency of $60 \mathrm{~Hz}$. Care must be taken to correctly interpret the angle in the presence of aliasing.

All the computed examples of this paper are half wave symmetric. Half wave symmetry means that the system states are equal in magnitude and opposite in sign to the system states half period or $T / 2$ later. Moreover, the firing times $\phi_{0}$ and $\phi_{1 / 2}$ and the thyristor switch off times $\tau_{0}$ and $\tau_{1 / 2}$ differ by exactly half a period.

\section{Open loop response}

This section compares the effects of synchronization with respect to zeros of TCSC voltage and line current by computing the dymamic response and eigenvalues as $\sigma$ varies [8].

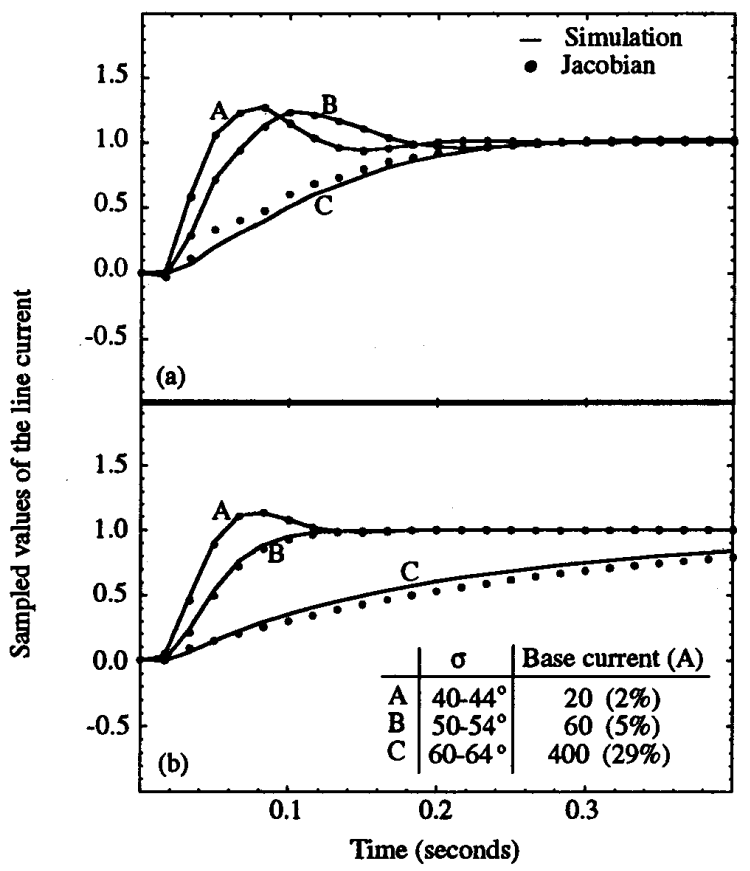

Figure 3. Response of line current to $4^{\circ}$ step change in $\sigma$ with synchronization on (a) voltage (b) line current.

The Kayenta system is used for all examples. The dynamic open loop response for both voltage and current 
synchronization are shown in Figure 3. The solid lines represent the solution of the state equations for successive sample points. A detailed EMTP simulation was done to confirm these results. The dots are derived by using the Jacobian to compute the dynamic response of the transfer function. The Jacobian computation closely approximates the simulated dynamic response. Figure 3 clearly shows the dependence of the dynamics on $\sigma$. The data using voltage synchronization agree with other published results [1].

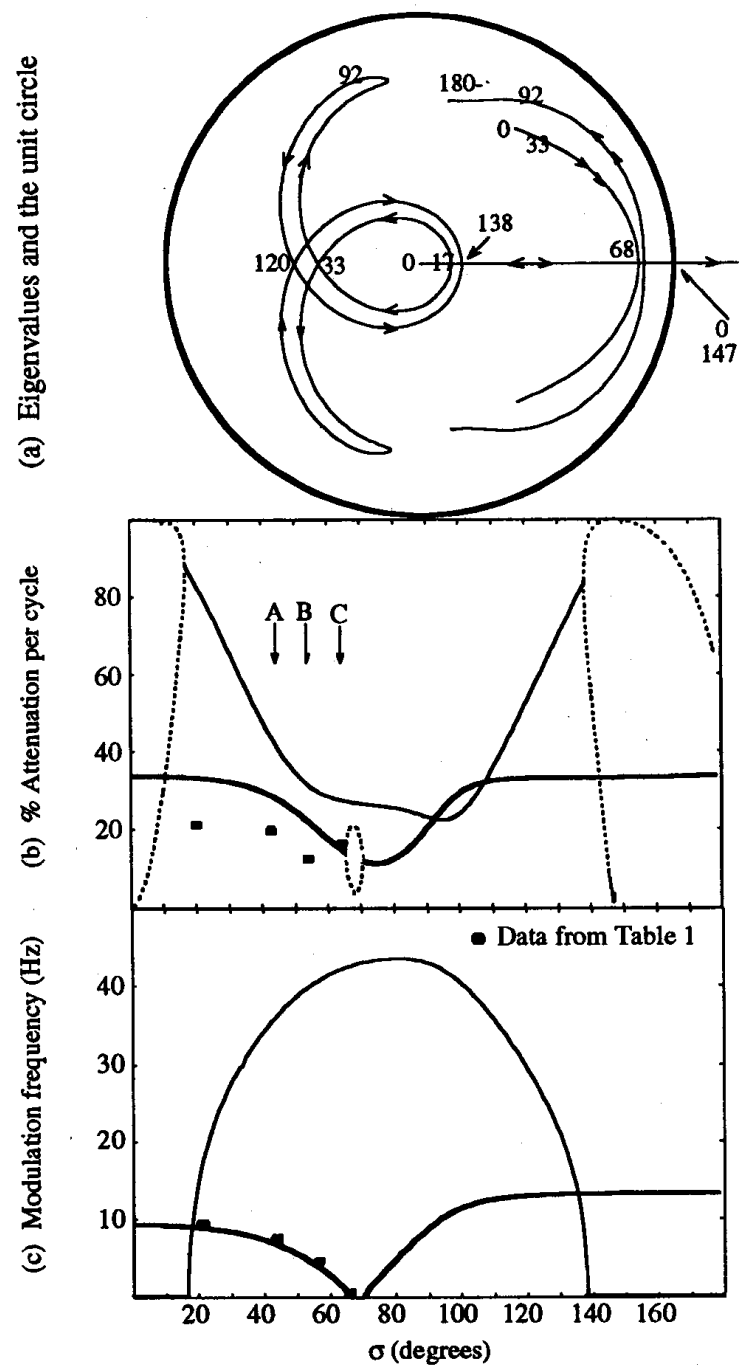

Figure 4. Eigenvalues for voltage synchronization. (a) locus of eigenvalues as $\sigma$ varies (b) Attenuation; real pole=---- , complex pole $=-$ (c) Modulation frequency.

Figure 4 shows the details of the calculated eigenvalues when the firing is synchronized to zeros of the voltage across the TCSC. The system has four eigenvalues. For some values of $\sigma$ these eigenvalues form complex pairs while at other values they lie on the real axis. This is best seen in figure $4(\mathrm{a})$ and (b). The letters $A, B, C$ indicate the values of $\sigma$ used in Figure 3. The dynamics calculated in [1] by fitting a fourth order transfer function are also shown as rectangles in Figure 4 . The differences may be due to the fitting methods used or the inclusion of filters or other dynamical components in the modeling.

Note that one of the eigenvalues leaves the unit circle for values of $\sigma$ greater than $140^{\circ}$ which implies that the halfwave symmetric solution becomes unstable. EMTP simulations verify this fact. (More detailed analysis shows that the stability is lost by an eigenvalue of the Jacobian of the halfwave map leaving the unit circle at -1 in a period doubling bifurcation.)

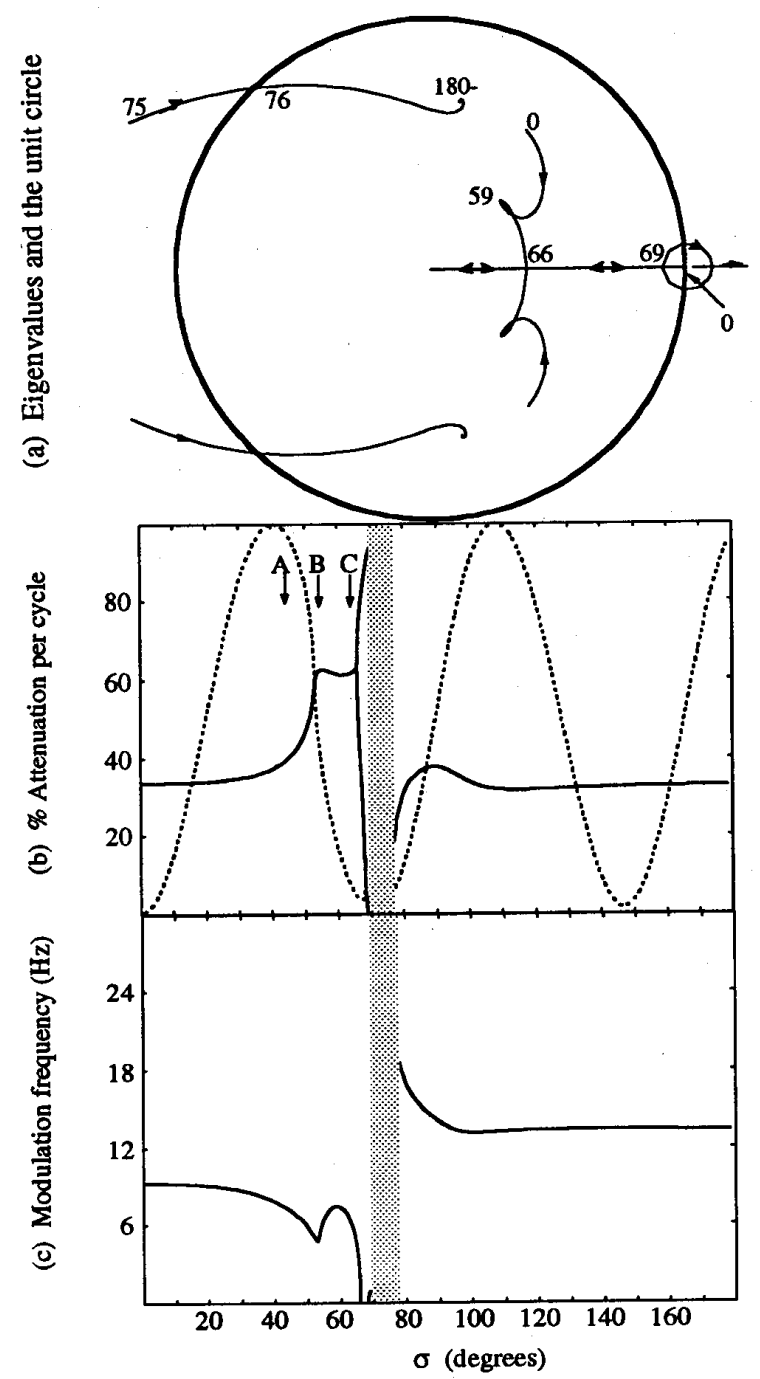

Figure 5. Eigenvalues for current synchronization. (a) locus of eigenvalues as $\sigma$ varies (b) Attenuation; real pole $=. . .$. , complex pole $=-$ (c) Modulation frequency. 
Figure 5 shows the details of the calculated eigenvalues when the firing is synchronized to zeros of the line current. This synchronization was used in the final controls for Kayenta. In general the line current is very stable and less susceptible to ambient harmonics. The half wave symmetric steady state is unstable for $\sigma$ between $69^{\circ}$ and $76^{\circ}$ and is shown as a gray area in Figure 5. The differences in the eigenvalues of Figures 4 and 5 show the significant differences in dynamic response between voltage and current synchronizations.

\section{Closed loop control}

A basic control issue for TCSC is the dependence of the dynamic response on the operating point of $\sigma$; see Figure 3. To reduce this variance, a feedback control on $\sigma$ is proposed. In this controller the error function is the difference between a requested $\sigma_{r e q}$ and a measured value $\sigma_{m}$. The measured value $\sigma_{m}$ is updated twice per period while the requested value can change continuously depending on the action of higher level controllers. The controller is a PI controller as shown in Figure 6. The controller gains $K_{p}=-10 \operatorname{Exp}\left[-\sqrt{\left(65-\sigma_{\text {req }}\right) / 2}\right]$ and $K_{i}=-23-24 \operatorname{Exp}\left[\left(\sigma_{r e q}-65\right) / 2\right]$ were chosen to develop a uniform response over the TCSC impedance ranging from 1.0 to 3.0 p.u. capacitive, or $\sigma$ less than $64^{\circ}$ (the normal capacitive range of operation).

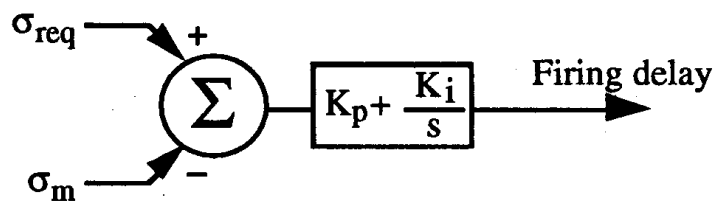

Figure 6. $\sigma$ Controller.

The formulation of the Poincare map of the closed loop system and the computation of its Jacobian are described in Appendix D. For this example, line current synchronization was assumed and two extra states were added to represent the control.

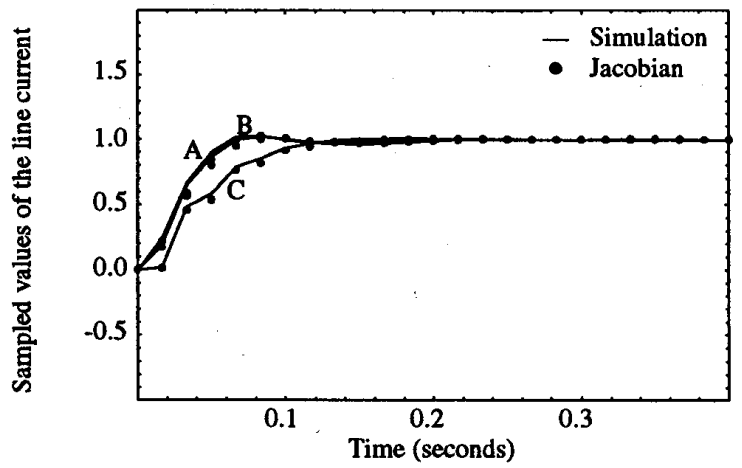

Figure 7. Closed loop system response.
The dynamic response to step changes in $\sigma$ is shown in Figure 7. The step changes in $\sigma$ are the same as for the open loop case. The dymamics are greatly improved when compared to the open loop response shown in Figure $3 \mathrm{~b}$. The dynamics are almost uniform across the operating range of $\sigma$ and overshoots and undershoots are greatly reduced. The transients settle in half the time required in the open loop cases.

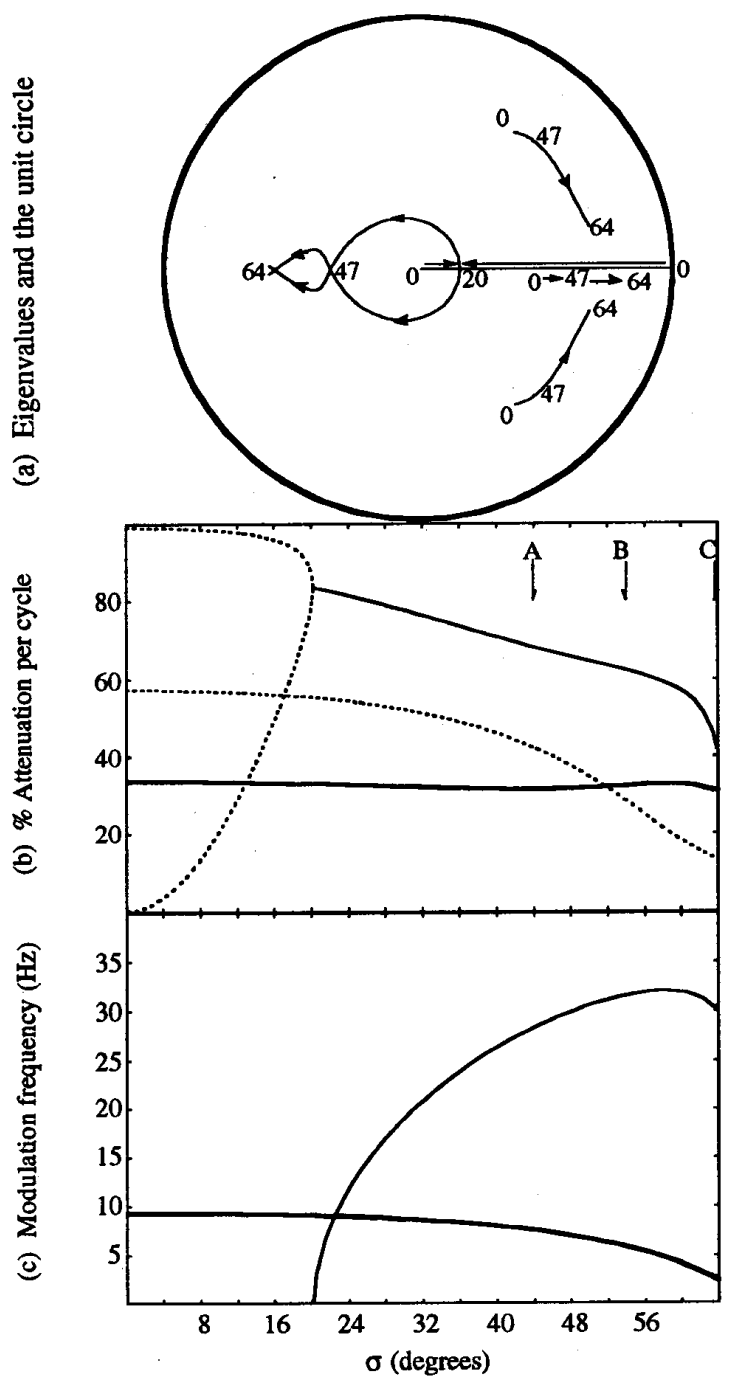

Figure 8. Eigenvalues of the closed loop system. (a) locus of eigenvalues as $\sigma$ varies (b) Attenuation; real pole $=. . .$. , complex pole $=-$ (c) Modulation frequency.

The closed loop eigenvalues are shown in Figure 8. In this example, the operation of TCSC was limited to the capacitive region, or $\sigma$ less than $64^{\circ}$. Compared to the open loop response in Figure 5 there are important differences. Comparing the dotted curves in Figure 5 (b) and Figure 8 (b) shows a marked difference in the damp- 
ing near $64^{\circ}$. In the open loop case, the damping has an oscillatory behavior showing very low attenuation close to $64^{\circ}$ whereas in the closed loop case, the attenuation is much more uniform. The modulation frequencies also show more uniform behavior for the closed loop case.

\section{Conclusion}

The effects of synchronization and thyristor switching on system dynamics have traditionally been difficult to include in models for control design. Detailed and time consuming simulations have been used to approximate transfer functions for the control design at a selection of operating points. This paper derives formulas for the linearized dynamics of a TCSC system with synchronization and a feedback control. Evaluating the formulas yields the system Jacobian and eigenvalues as a function of firing angle $\sigma$ which can then be used in controller design. It is more insightful and much quicker to evaluate formulas for a general operating point than to numerically approximate transfer functions with simulations. Currently the authors are investigating the applications of these methods to SSR damping using TCSC, the design of SVC compensators and HVDC controllers.

\section{Acknowledgment}

The authors gratefully acknowledge funding in part from EPRI under contracts RP 4000-29 and RP 8010-30 and from NSF PYI grant ECS-9157192.

\section{References}

[1] N. Christl, R.Hedin, K. Sadek et al., "Advanced series compensation (ASC) with thyristor controlled impedance", CIGRÉ 14/37/38-05, Aug. 1992.

[2] R. Johnson, P. Krause, A. Montoya, N. Christl, R. Hedin, "Power system studies and modeling for the Kayenta $230 \mathrm{kV}$ substation advanced series compensation", IEE Fifth Intl. Conf. on AC and DC Power transmission, Sept. 1991, London UK.

[3] J.P. Louis, "Non-linear and linearized models for control systems including static convertors", Third Intl. Federation on Automatic Control Symp. on Control in power electronics and electrical drives, Lausanne, Switzerland, Sept. 1983 pp 9-16.

[4] M. Grötzbach, R. von Lutz, "Unified modeling of rectifier-controlled DC-power supplies", IEEE Trans. on Power Electronics, Vol. 1, No. 2, April 1986, pp. 90-100.

[5] G.C. Verghese, M.E. Elbuluk, J.G. Kassakian, "A general approach to sampled-data modeling for power electronic circuits", IEEE Trans. on Power Electronics, Vol. 1, No. 2, April 1986, pp. 76-89.

[6] S.G. Jalali, I. Dobson, R.H.Lasseter, "Instabilities due to bifurcation of switching times in a thyristor controlled reactor", Power Electronics Specialists Conf., Toledo, Spain, July 1992, pp. 546-552.

[7] I. Dobson, S.G. Jalali, R. Rajaraman, "Damping and Resonance in a High Power Switching Circuit",
Institute for Math and its Applications workshop paper, March 1993, to appear in Systems and Control Theory for Power Systems (eds. J. Chow, P. Kokotovic, R.J. Thomas), IMA volumes in mathematics and its applications, Springer Verlag.

[8] S.G. Jalali, "Harmonics and Instabilities in Thyristor Based Switching Circuits", Ph.D. Thesis, University of Wisconsin at Madison, 1993.

[9] J.M.T. Thompson, H.B. Stewart, Nonlinear Dynamics and chaos: geometrical methods for scientists and engineers, John Wiley, London, 1987.

[10] I. Dobson, S.G. Jalali, "Surprising simplification of the Jacobian of a diode switching circuit", IEEE Intl. Symp. on Circuits and Systems, Chicago, IL, May 1993, pp. 2652-2655.

[11] R. Rajaraman, I. Dobson, S.G. Jalali, "Nonlinear dynamics and switching time bifurcations of a thyristor controlled reactor", IEEE Intl. Symp. on Circuits and Systems, Chicago, IL, May 1993, pp. 2180-2183.

\section{Appendix A. System Modeling}

This section describes the system modeling with thyristor firing synchronized with voltage or current zeros. During the thyristor conduction time, the system state vector is $x(t)=\left(I_{r}(t), V_{r}(t), I_{s}(t), V_{s}(t)\right)^{t}$, where $I_{r}$ is the thyristor current, $V_{r}$ is the thyristor controlled capacitor voltage, $I_{s}$ is the line current and $V_{s}$ is the fixed capacitor voltage. The system dynamics are described by the linear differential equations:

$$
\dot{x}=A x+B u
$$

where

$$
A=\left(\begin{array}{cccc}
0 & L_{r}^{-1} & 0 & 0 \\
-C_{r}^{-1} & 0 & C_{r}^{-1} & 0 \\
0 & L_{s}^{-1} & -R_{s} L_{s}^{-1} & -L_{s}^{-1} \\
0 & 0 & C_{s}^{-1} & 0
\end{array}\right), B=\left(\begin{array}{c}
0 \\
0 \\
L_{s}^{-1} \\
0
\end{array}\right)
$$

, $L_{s}=406 \mathrm{mH}$ and $R_{s}=19.89 \Omega$ are the total line impedance and resistance, $C_{s}=27.9 \mu \mathrm{F}$ is the fixed capacitor, $L_{r}=6.8 \mathrm{mH}$ and $C_{r}=177 \mu \mathrm{F}$ are the thyristor controlled reactor and capacitor, and the net source voltage $u(t)=67 \sin \omega t \mathrm{kV}$. During the off time of each thyristor, $I_{r}$ is identically zero and the system state vector is $y(t)=\left(V_{c}(t), I_{s}(t), V_{s}(t)\right)^{t}$ and the system dynamics are

$\dot{y}=P A P^{t} y+P B u$
where $P$ is the projection matrix $P=\left(\begin{array}{llll}0 & 1 & 0 & 0 \\ 0 & 0 & 1 & 0 \\ 0 & 0 & 0 & 1\end{array}\right)$.

The circuit state at the turn on time $\phi_{0}$ is denoted either by $y\left(\phi_{0}\right)$ or by $x\left(\phi_{0}\right)$ and these are related by

$$
x\left(\phi_{0}\right)=P^{t} y\left(\phi_{0}\right)
$$

The state at the turn off time $\tau_{0}$ is denoted either by $x\left(\tau_{0}\right)$ or $y\left(\tau_{0}\right)$ and these are related by

$$
y\left(\tau_{0}\right)=P x\left(\tau_{0}\right)
$$


The thyristor turn on times at $\phi_{1 / 2}$ and $\phi_{1}$ depend on the firing scheme:

(a) Synchronizing the firing with respect to the zeros of the voltage $V_{r}$ is given by

$$
\phi_{1 / 2}=\tau_{v 0}+\pi-\sigma_{\text {req }} / 2
$$

where $\tau_{v 0}$ satisfies $V_{r}\left(\tau_{v 0}\right)=0$ and $\sigma_{r e q}$ is the requested value of $\sigma . \tau_{v 0}$ is assumed to occur when the thyristor is conducting.

(b) Synchronizing the firing with respect to the zeros of the line current $I_{s}$ is given by

$$
\phi_{1 / 2}=\tau_{c 0}+\left(\pi-\sigma_{r e q}\right) / 2
$$

where $\tau_{c 0}$ satisfies $I_{s}\left(\tau_{c 0}\right)=0 . \tau_{c 0}$ is assumed to occur when both the thyristor are off.

\section{Appendix B. Poincaré map}

This section sketches the construction of a Poincaré map as in $[6,10,8]$. Given a time interval $\left[s_{1}, s_{2}\right]$, it is convenient to write $f\left(x, s_{1}, s_{2}\right)$ for the map which advances the state $x\left(s_{1}\right)$ at $s_{1}$ to the state $x\left(s_{2}\right)$ at $s_{2}$. If the thyristor is on during all of the time interval [ $s_{1}, s_{2}$ ], we write $f\left(x, s_{1}, s_{2}\right)$ as $f_{\text {on }}\left(x, s_{1}, s_{2}\right)$. Similarly, if the thyristor is off during all of $\left[s_{1}, s_{2}\right]$, we write $f\left(y, s_{1}, s_{2}\right)$ as $f_{\text {off }}\left(y, s_{1}, s_{2}\right)$. A half period map advancing the state from $t_{0}$ to $t_{1 / 2}$ may be written in terms of $f_{\text {on }}$ and $f_{\text {off }}$ taking into account the coordinate changes (A3) or (A4) at the switching times:

$$
\begin{aligned}
& f\left(x_{0}, t_{0}, t_{1 / 2}\right)= \\
& f_{\text {on }}\left(P^{t} f_{\text {off }}\left(P f_{\text {on }}\left(x_{0}, t_{0}, \tau_{0}\right), \tau_{0}, \phi_{1 / 2}\right), \phi_{1 / 2}, t_{1 / 2}\right)
\end{aligned}
$$

The Poincaré map $F$ may now be written by composing two successive half period maps and then neglecting the details of the time arguments:

$$
\begin{aligned}
F\left(x_{0}\right) & =f\left(x_{0}, t_{0}, t_{0}+T\right) \\
& =f\left(f\left(x_{0}, t_{0}, t_{1 / 2}\right), t_{1 / 2}, t_{1}\right) \\
& =f_{\text {on }} P^{t} f_{\text {off }} P f_{\text {on }} P^{t} f_{\text {off }} P f_{\text {on }}\left(x_{0}\right)
\end{aligned}
$$

\section{Appendix C. Computation of the Jacobian}

This section derives the formulas to compute the Jacobian $D F$. Since the thyristor turn off time and the Poincaré map are discontinuous at a switching time bifurcation, we assume that the system is not at a switching time bifurcation $[6,11,8]$. The first step is to compute the Jacobian of the half period map $f\left(x_{0}, t_{0}, t_{1 / 2}\right)$. Define $H_{0}\left(x_{0}, r_{0}, \phi_{1 / 2}\right)$ to be the right hand side of (B1). $H_{0}$ expresses $x\left(t_{1 / 2}\right)$ as a function of $x_{0}$, the turn off time $\tau_{0}$ and the turn on time $\phi_{1 / 2} . \tau_{0}$ is a function of $x_{0}$ which is determined by the constraint of zero thyristor current at time $\tau_{0}$. The turn on time $\phi_{1 / 2}$ depends on $x_{0}$ via the firing strategy. The half period map may be written as

$$
f\left(x_{0}, t_{0}, t_{1 / 2}\right)=H_{0}\left(x_{0}, \tau_{0}\left(x_{0}\right), \phi_{1 / 2}\left(x_{0}\right)\right)
$$

By the chain rule, the Jacobian of the half period map is:

$$
D f\left(x_{0}, t_{0}, t_{1 / 2}\right)=\frac{\partial H_{0}}{\partial x_{0}}+\frac{\partial H_{0}}{\partial \tau_{0}} D \tau_{0}+\frac{\partial H_{0}}{\partial \phi_{1 / 2}} D \phi_{1 / 2}
$$

Now we compute the partial derivatives of $H_{0}$ in (C1).

$$
\frac{\partial H_{0}}{\partial x_{0}}=e^{A\left(t_{1 / 2}-\phi_{1 / 2}\right)} P^{t} e^{P A P^{t}\left(\phi_{1 / 2}-\tau_{0}\right)} P e^{A\left(\tau_{0}-t_{0}\right)} .
$$

since the only term of $H_{0}\left(x_{0}, \tau_{0}, \phi_{1 / 2}\right)$ depending on $x_{0}$ is the right hand side of (C2) times $x_{0}$. An important simplification proved in $[3,10]$ and also used in $[6,7]$ asserts that

$$
\frac{\partial H_{0}}{\partial \tau_{0}}=0
$$

$H_{0}\left(x_{0}, \tau_{0}, \phi_{1 / 2}\right)$ can also be written as

$$
e^{A\left(t_{1 / 2}-\phi_{1 / 2}\right)}\left[P^{t} y\left(\phi_{1 / 2}\right)+\int_{\phi_{1 / 2}}^{t_{1 / 2}} e^{A\left(\phi_{1 / 2}-s\right)} B u(s) d s\right]
$$

and differentiating and using (A1) gives

$$
\frac{\partial H_{0}}{\partial \phi_{1 / 2}}=e^{A\left(t_{1 / 2}-\phi_{1 / 2}\right)}\left[P^{t} \dot{y}\left(\phi_{1 / 2}-\right)-\dot{x}\left(\phi_{1 / 2}+\right)\right]
$$

(The notation $y\left(\phi_{1 / 2}-\right)$ means the limit of $y(t)$ as $t$ approaches $\phi_{1 / 2}$ from below.) Since (A2), (A4) and (A1) imply that $\dot{y}\left(\phi_{1 / 2}-\right)=P \dot{x}\left(\phi_{1 / 2}+\right)$ and the form of $P$ implies that $P^{t} P-I=-c^{t} c$, where $c=(1,0,0,0),(\mathrm{C} 4)$ may be rewritten as

$$
\frac{\partial H_{0}}{\partial \phi_{1 / 2}}=-e^{A\left(t_{1 / 2}-\phi_{1 / 2}\right)} c^{t} c \dot{x}\left(\phi_{1 / 2}+\right)
$$

Note that $c \dot{x}\left(\phi_{1 / 2}+\right)$ is the gradient of the thyristor current as it turns on at $\phi_{1 / 2}$.

Now we compute the term $D \phi_{1 / 2}$ in (C1). The row vector $D \phi_{1 / 2}$ is the gradient of the turn on time $\phi_{1 / 2}$ with respect to $x_{0} . D \phi_{1 / 2}$ depends on the firing scheme:

(a) For voltage synchronization, differentiation of (A5) yields $D \phi_{1 / 2}=D \tau_{v 0}$ so that we need to compute $D \tau_{v 0}$. The constraint determining $\tau_{v 0}$ is $0=V_{r}\left(\tau_{v 0}\right)=$ $m x\left(\tau_{v 0}\right)=m f_{\text {on }}\left(x_{0}, t_{0}, \tau_{v 0}\right)$ where $m=(0,1,0,0)$. Differentiation with respect to $x_{0}$ yields $0=m D f_{\text {on }}+$ $\left.m \frac{\partial f_{o n}}{\partial \tau_{v 0}}\right|_{\tau_{v 0}} D \tau_{v 0}=m e^{A\left(\tau_{v 0}-t_{0}\right)}+m \dot{x}\left(\tau_{v 0}\right) D \tau_{v 0}$ and

$$
D \phi_{1 / 2}=D \tau_{v 0}=-m e^{A\left(\tau_{v 0}-t_{0}\right)} / m \dot{x}\left(\tau_{v 0}\right)
$$

Note that $m \dot{x}\left(\tau_{v 0}\right)$ is the gradient of $V_{r}$ as it passes through zero at $\tau_{v 0}$.

(b) For current synchronization, differentiation of (A6) yields $D \phi_{1 / 2}=D \tau_{c 0}$. The constraint determining $\tau_{c 0}$ is $0=I_{s}\left(\tau_{c 0}\right)=n y\left(\tau_{c 0}\right)$ where $n=(0,1,0)$. Differentiating the constraint as in case (a) yields

$$
\begin{gathered}
D \phi_{1 / 2}=D \tau_{c 0}=-n D f\left(x_{0}, t_{0}, \tau_{c 0}\right) / n \dot{y}\left(\tau_{c 0}\right) \\
=-n e^{P A P^{t}\left(\tau_{c 0}-\tau_{0}\right)} P e^{A\left(\tau_{c 0}-t_{0}\right)} / n \dot{y}\left(\tau_{c 0}\right)
\end{gathered}
$$

where the final expression for $D f\left(x_{0}, t_{0}, \tau_{c 0}\right)$ was obtained using the simplification (C3). 
The corresponding equations for half period map $f\left(x_{1 / 2}, t_{1 / 2}, t_{1}\right)$ can be obtained from the results above by changing all of the subscripts ' $1 / 2$ ' to ' 1 ' and all of the subscripts ' 0 ' to ' $1 / 2$ '. Finally, differentiating (B2) and using the chain rule, the Jacobian of the Poincaré map is

$$
D F=D f\left(x_{1 / 2}, t_{1 / 2}, t_{1}\right) D f\left(x_{0}, t_{0}, t_{1 / 2}\right)
$$

If the steady state waveform is assumed to be half wave symmetric, then (C8) simplifies to

$$
D F=\left(\frac{\partial H_{0}}{\partial x_{0}}+\frac{\partial H_{0}}{\partial \phi_{1 / 2}} D \phi_{1 / 2}\right)^{2}
$$

or, in detail for the case of voltage synchronization,

$$
\begin{aligned}
& D F=\left(e^{A\left(t_{1 / 2}-\phi_{1 / 2}\right)} P^{t} e^{P^{t} A P\left(\phi_{1 / 2}-\tau_{0}\right)} P e^{A\left(\tau_{0}-t_{0}\right)}\right. \\
& \left.+e^{A\left(t_{1 / 2}-\phi_{1 / 2}\right)} c^{t} c \dot{x}\left(\phi_{1 / 2}+\right) \frac{m e^{A\left(\tau_{v 0}-t_{0}\right)}}{m \dot{x}\left(\tau_{v 0}\right)}\right)^{2}
\end{aligned}
$$

In the case of current synchronization, one eigenvalue of $D F$ is identically zero.

\section{Appendix D. Closed loop control}

The controller equations can be written by inspecting figs. 2 and 6 . The error function $\epsilon$ is updated at each thyristor switch off. The error function at time $t_{1 / 2}$ is

$$
\epsilon_{1 / 2}=\sigma_{\text {req }}-\left(\tau_{0}-\phi_{0}\right)
$$

where $\phi_{0}$ can be computed from $x_{0}$. The integrator output $a$ at time $t_{1 / 2}$ is

$$
\begin{array}{r}
a_{1 / 2}=a_{0}+K_{i} \epsilon_{0}\left(\tau_{0}-t_{0}\right)+K_{i} \epsilon_{1 / 2}\left(t_{1 / 2}-\tau_{0}\right) \\
=a_{0}+K_{i} \epsilon_{0}\left(\tau_{0}-t_{0}\right)+K_{i}\left(\sigma_{\text {req }}+\phi_{0}-\tau_{0}\right)\left(t_{1 / 2}-\tau_{0}\right)(
\end{array}
$$

The midperiod turn on time $\phi_{1 / 2}$ depends on $\tau_{c 0}$ and the control output at time $\phi_{1 / 2}$ :

$$
\phi_{1 / 2}=\tau_{c 0}+K_{p} \epsilon_{1 / 2}+a_{0}+K_{i} \epsilon_{0}\left(\tau_{0}-t_{0}\right)+K_{i} \epsilon_{1 / 2}\left(\phi_{1 / 2}-\tau_{0}\right)
$$

and solving for $\phi_{1 / 2}$ and substituting for $\epsilon_{1 / 2}$ from (D1) gives

$$
\begin{aligned}
& \quad \phi_{1 / 2}= \\
& \frac{\tau_{c 0}+\left(K_{p}-K_{i} \tau_{0}\right)\left(\sigma_{r e q}+\phi_{0}-\tau_{0}\right)+a_{0}+K_{i} \epsilon_{0}\left(\tau_{0}-t_{0}\right)}{1-K_{i}\left(\sigma_{r e q}+\phi_{0}-\tau_{0}\right)}
\end{aligned}
$$

The circuit state at time $t_{1 / 2}$ is given by

$$
x_{1 / 2}=H_{0}\left(x_{0}, \tau_{0}\left(x_{0}\right), \phi_{1 / 2}\left(x_{0}, a_{0}, \epsilon_{0}\right)\right)
$$

The circuit state $x$ is augmented by the integrator output and the error to form a 6 dimensional state vector $z=(x, a, \epsilon)^{t}$. The equations for the half wave map $g\left(z_{0}, t_{0}, t_{1 / 2}\right)$ advancing the state $z_{0}=\left(x_{0}, a_{0}, \epsilon_{0}\right)^{t}$ to the state $z_{1 / 2}=\left(x_{1 / 2}, a_{1 / 2}, \epsilon_{1 / 2}\right)^{t}$ are (D4), (D2) and (D1). Differentiating these equations, using the simplification (C3), and omitting terms which vanish because $\epsilon_{0}=\epsilon_{1 / 2}=0$ when $D_{z_{0}} g$ is evaluated at the fixed point gives

$$
\begin{gathered}
D_{z_{0}} g\left(z_{0}, t_{0}, t_{1 / 2}\right)= \\
\left(\begin{array}{ccc}
\frac{\partial H_{0}}{\partial x_{0}}+\frac{\partial H_{0}}{\partial \phi_{1 / 2}} D \phi_{1 / 2} & \frac{\partial H_{0}}{\partial \phi_{1 / 2}} & \frac{\partial H_{0}}{\partial \phi_{1 / 2}} K_{i}\left(\tau_{0}-t_{0}\right) \\
K_{i}\left(D \phi_{0}-D \tau_{0}\right)\left(t_{1 / 2}-\tau_{0}\right) & 1 & K_{i}\left(\tau_{0}-t_{0}\right) \\
D \phi_{0}-D \tau_{0} & 0 & 0
\end{array}\right)
\end{gathered}
$$

where $\frac{\partial H_{0}}{\partial x_{0}}$ and $\frac{\partial H_{0}}{\partial \phi_{1 / 2}}$ are given by (C2) and (C5) and the term $D \phi_{1 / 2}$ evaluates to

$$
D \phi_{1 / 2}=D \tau_{c 0}+\left(K_{p}+K_{i}\left(\tau_{c 0}+a_{0}-\tau_{0}\right)\right)\left(D \phi_{0}-D \tau_{0}\right)
$$

In (D6), $D \tau_{c 0}$ is given by (C6b) and it remains to compute $D \phi_{0}-D \tau_{0}$. The constraint equation determining $\tau_{0}$ is $0=I_{r}\left(\tau_{0}\right)=c x\left(\tau_{0}\right)=c f_{\text {on }}\left(x_{0}, t_{0}, t_{0}+\tau_{0}\right)$, where $c=(1,0,0,0)$. Differentiation with respect to $x_{0}$ yields $0=c D f_{\text {on }}+\left.c \frac{\partial f_{\text {on }}}{\partial \tau_{0}}\right|_{\tau_{0}} D \tau_{0}=c e^{A\left(\tau_{0}-t_{0}\right)}+c \dot{x}\left(\tau_{0}-\right) D \tau_{0}$ and $D \tau_{0}=-c e^{A\left(\tau_{0}-t_{0}\right)} / c \dot{x}\left(\tau_{0}-\right)$. Note that $c \dot{x}\left(\tau_{0}-\right)$ is the gradient of the thyristor current as it turns off at $\tau_{0}$. Similarly, $D \phi_{0}=-c e^{A\left(\phi_{0}-t_{0}\right)} / c \dot{x}\left(\phi_{0}+\right)$ and hence

$D \phi_{0}-D \tau_{0}=-c e^{A\left(\tau_{0}-t_{0}\right)} / c \dot{x}\left(\tau_{0}-\right)+c e^{A\left(\phi_{0}-t_{0}\right)} / c \dot{x}\left(\phi_{0}+\right)$

The equations for the half wave map $g\left(z_{1 / 2}, t_{1 / 2}, t_{1}\right)$ and its Jacobian $D_{z_{1 / 2}} g\left(z_{1 / 2}, t_{1 / 2}, t_{1}\right)$ can be transcribed from the corresponding results above by changing all of the subscripts ' $1 / 2$ ' to ' 1 ' and all of the subscripts ' 0 ' to ' $1 / 2$ '. The Poincaré map $G\left(z_{0}\right)=$ $g\left(g\left(z_{0}, t_{0}, t_{1 / 2}\right), t_{1 / 2}, t_{1}\right)$ and finally the Poincaré map Jacobian can be computed from

$$
D_{z_{0}} G=D_{z_{1 / 2}} g\left(z_{1 / 2}, t_{1 / 2}, t_{1}\right) D_{z_{0}} g\left(z_{0}, t_{0}, t_{1 / 2}\right)
$$

We remark that one eigenvalue of $D_{z_{0}} G$ is identically zero and that (D7) does not simplify under half wave symmetry.

Sasan Jalali (S'92) received the BS, MS and PhD in Electrical Engineering in 1988, 1990 and 1993 from the University of Wisconsin-Madison. His interests include application of power electronics to utility systems

Robert H. Lasseter (F'92) received the $\mathrm{PhD}$ degree in Physics at the University of Pennsylvania, Philadelphia in 1971. He was a Consultant Engineer at General Electric Company until he joined the University of Wisconsin-Madison in $\mathbf{1 9 8 0}$. His main interest is the application of power electronics to utility systems.

Ian Dobson (M'89) received the PhD in Electrical Engineering from Cornell University and joined the University of Wisconsin-Madison faculty in 1989. His current interests are bifurcations, nonlinear dynamics, voltage collapse and switching circuits. 